\title{
Practical estimation of the uncertainty of analytical measurement standards
}

\author{
Ruud J. B. Peters $\cdot$ Ingrid J. W. Elbers \\ Mirjam D. Klijnstra · Alida A. M. Stolker
}

Received: 24 May 2011/Accepted: 30 August 2011/Published online: 16 September 2011

(C) The Author(s) 2011. This article is published with open access at Springerlink.com

\begin{abstract}
Nowadays, a lot of time and resources are used to determine the quality of goods and services. As a consequence, the quality of measurements themselves, e.g., the metrological traceability of the measured quantity values is essential to allow a proper evaluation of the results with regard to specifications and regulatory limits. This requires knowledge of the measurement uncertainties of all quantity values involved in the measurement procedure, including measurement standards. This study shows how the uncertainties due to the preparation, as well as the chemical and compositional stability of a chemical measurement standard, or calibrator, can be estimated. The results show that the relative standard uncertainty of the concentration value of a typical analytical measurement standard runs up to $2.8 \%$ after 1 year. Of this, $1.9 \%$ originates from the preparation of the measurement standard, while 2.0 and $0.53 \%$ originate from the chemical and compositional stability during storage at $-20{ }^{\circ} \mathrm{C}$. The monthly preparation of working calibrators stored at $4{ }^{\circ} \mathrm{C}$ and used on a weekly basis, results in an additional standard uncertainty of the analyte concentration value of $0.35 \%$ per month due to compositional stability. While the preparation procedure is the major contributor to the total measurement uncertainty, the uncertainties introduced by the stability measurements are another important contributor, and therefore, the measurement procedure to evaluate stability is important to minimize the total measurement uncertainty.
\end{abstract}

R. J. B. Peters $(\varangle)$ · I. J. W. Elbers · M. D. Klijnstra

A. A. M. Stolker

RIKILT-Institute of Food Safety, Wageningen UR,

Akkermaalsbos 2, P.O. Box 230,

6700 AE Wageningen, The Netherlands

e-mail: ruudj.peters@wur.nl
Keywords Metrological traceability .

Measurement and testing · Quality assurance .

Measurement uncertainty · Analytical

measurement standards · Chemical stability

\section{Introduction}

During the last half of the previous century, the focus on the quality of goods and services increased. This implied a requirement that components, raw materials and finished goods, including food and drugs, should meet quality and regulatory standards. As a consequence, the quality of measurements that form the basis of commercial and regulatory decisions became an important topic. The concept, on which this is built, is known as metrological traceability and is an important general concept in measurement. For analytical laboratories, it requires that the uncertainty of analytical measurement is evaluated, that detection and decision levels like $\mathrm{CC} \beta$ and $\mathrm{CC} \alpha$ values include the measurement uncertainty, or even that individually reported analytical results are accompanied by their measurement uncertainty. In this context, the International Organization for Standardization (ISO) produced three important documents: ISO/IEC 17025:2005, "General requirements for the competence of calibration and testing laboratories" [1]; "Guide to the expression of uncertainty in measurement", commonly referred to as GUM [2]; and ISO21748, "Guidance for the use of repeatability, reproducibility and trueness estimates in measurement uncertainty estimation" [3]. In addition, Eurachem and CITAC published a guide how to determine the measurement uncertainty of a result in chemical analysis [4], and more recently, a guide describing the use of uncertainty information in compliance testing, such as testing for the presence of veterinary drugs in food or doping in sports [5]. Other practical 
and simplified approaches for the evaluation of measurement uncertainty can be found in the literature [6-10]. This study focuses on the measurement uncertainty of the analyte concentration value carried by the analytical measurement standard, or chemical calibrator, used in the analysis of contaminants and residues. It identifies methods and procedures how this uncertainty can be evaluated and how it contributes to the total measurement uncertainty of a measurement result.

The uncertainty in the analyte concentration value of a chemical calibrator refers to two distinctly different uncertainty components: uncertainty in the analyte concentration due to the preparation of the calibrator, $u$ (prep), and possible changes in the analyte concentration during storage and/or use, $u$ (shelf), as described in Eq. 1.

$u^{2}($ calibrator $)=u^{2}($ prep $)+u^{2}($ shelf $)$

It is important to notice that the uncertainties associated with analyte concentration and possible degradation do not necessarily reflect true deviations or degradation. These uncertainties are not zero because of the uncertainties associated with the instruments used to prepare chemical calibrators and the uncertainties introduced by the measurement of potential degradation. Unfortunately, in general little effort is spent in the assessment of the contribution of individual sources like the analytical measurement standards to the total uncertainty of a measurement procedure. In addition, the approaches for the evaluation of measurement uncertainty found in the literature show that the definition and use of concepts involved in metrological traceability is insufficient and varied. The "International Vocabulary of MetrologyBasic and general concepts and associated terms", shortly referred to as VIM [11], and a publication by De Bièvre [12], provide a basis for the vocabulary that will be followed in this study.

\section{Materials and methods}

The combined standard uncertainty $u(c)$ of the mass concentration value, $c$, of an analyte in an analytical measurement standard depends upon all input quantities involved in the preparation process. Analysts can decrease the uncertainty by identifying all sources of uncertainty in the preparation process, understanding the principles of uncertainty propagation, and compiling an uncertainty budget. The uncertainty budget lists the sources of uncertainty and their associated standard uncertainties, compiled with a view to evaluating a combined standard uncertainty associated with a measurement result. Metrological traceability is a prerequisite for this evaluation and for the identification of the components of the budget. The general relationship between $u(c)$ and the uncertainty components is as follows:

$u(c)=\sqrt{\sum_{i=1, n} k_{i}^{2} u\left(x_{i}\right)^{2}}=\sqrt{\sum_{i=1, n} u\left(c, x_{i}\right)^{2}}$

where $x_{1}, x_{2}, \ldots, x_{n}$ are the individual components that determine the mass concentration $c, k_{i}$ is a sensitivity coefficient evaluated as $k_{i}=\delta c / \delta x_{i}$, the partial differential of $c$ with respect to $x_{i}$, and $u\left(c, x_{i}\right)$ denotes the uncertainty in $c$ arising from the uncertainty in $x_{i}$. The process of compiling the uncertainty budget is most properly divided into the following steps: (1) Specification of how the standard is prepared, (2) Identification of sources and quantification of their contribution to uncertainty, and (3) Calculation of the combined uncertainty. To calculate the total uncertainty, the uncertainty propagation law can be used for expressions in which there are only divisions and products. In that case all individual components are converted into relative uncertainties. From this the standard uncertainty and expanded uncertainty of any measurement standard or calibrator can be calculated as in example A1 presented in QUAM [4]. The concentration of an analyte in a chemical measurement standard can change in time due to a limited stability of the analyte itself (chemical stability) or the calibrator solution (compositional stability). This has been studied before by Linsinger et al. [13] for the stability of reference materials and can as well be used for measurement standards or calibrators. For any changes in the analyte concentration, they assumed that a straight line can be fitted through the data, i.e., it is assumed that the concentration value $c$ changes linearly from the initial value $c_{0}$ with a rate $b$ and after time $t$ as:

$c=c_{0}(1+b \cdot t)$

The rate $b$ is negative for decreasing and positive for increasing concentrations and can be approximated by the slope of the regression line. The most important conclusion that can be drawn from stability measurements is the presence or absence of a significant trend in the data that would hint at a change in the analyte concentration over time. If we assume a linear change in the concentration during storage or use of the standard, the uncertainty in the concentration of the calibrator $u(c)$ at any time can be estimated by propagating the uncertainties $u\left(c_{0}\right), u(t)$, and $u(b)$ of the variable $c$ to the independent variables $c_{0}, t$ and $b$, i.e., taking the partial derivatives of $c$. Since $u\left(c_{0}\right)$ is equal to the uncertainty of the calibrator preparation, i.e., $u$ (prep), $u\left(c_{0}\right)$ has nothing to do with the uncertainty in the stability of the analyte concentration value of the measurement standard. The derivative of $c$ with respect to $t$ is equal to $c_{0} b$ and this term corresponds to the real change of the analyte concentration in time. If the slope $b$ is not 
significant and set to zero, $c_{0} b$ is also zero. The derivative of $c$ with respect to $b$ is equal to $c_{0} t$ and this corresponds to the uncertainty in the measurement of the change rate $b$. With $u(b)$ being the standard uncertainty of the slope calculated from the standard deviation of all measurements, the uncertainty becomes $t u(b)$. If no change in the concentration is observed over time, i.e., $b=0, t u(b)$ can be used to estimate $u$ (shelf). This is in agreement with common sense since very close to $t=0, u$ (shelf) is negligible, however, the larger $t$ becomes, the less certain one is about the analyte concentration.

For stability testing of an analyte in solution, the Commission Decision 2002/657/EC [14] proposes that the analyte content should be measured in a freshly prepared measurement standard. Thereafter, 10 aliquots of the measurement standard should be stored in the dark at $-20{ }^{\circ} \mathrm{C},+4{ }^{\circ} \mathrm{C}$ and at $+20{ }^{\circ} \mathrm{C}$ and in daylight at $+20^{\circ} \mathrm{C}$. A storage time of 1-4 weeks, or longer until the first degradation phenomena are observed, should be used and the measurement standard should be analyzed at regular intervals to measure analyte degradation. While this method does measure changes in analyte concentration due to possible degradation of analytes during storage, i.e., chemical stability, it does not determine potential changes in composition as a consequence of the actual use of the measurement standard over a period of time. These changes in composition may be caused by the storage at higher temperatures $\left(4^{\circ} \mathrm{C}\right.$ or even room temperature), by the frequent changes in temperature (vials containing measurement standards are allowed to warm to room temperature before aliquots are collected), or by the mixing and actual opening of the vials containing the measurement standard. The combined effect of chemical stability and usage may be evaluated by measuring the concentration of the analyte in time. However, a different approach is proposed here. The most important conclusion that can be drawn from measuring analyte concentrations with time is the presence or absence of a significant trend in the measurement results that would hint at a change in the analyte concentration. The significance of this trend is determined using the Student's $t$ test by comparing the quotient of the slope and the uncertainty in the slope, $b / u(b)$, to the value of the $t$ statistics with a given confidence level. To be able to identify significant trends, it is important to minimize the uncertainty in the slope $u(b)$ by minimizing the variation in the stability measurements and by increasing the number of stability measurements. This can be achieved by analyzing all stability samples in one series, an approach sometimes referred to as isochronous [15]. Therefore, chemical stability is tested by the preparation of a stock solution that is divided over a number of vials that are stored at $-20{ }^{\circ} \mathrm{C}$ and $+4{ }^{\circ} \mathrm{C}$. After periods of $0,1,3,6,9$, and 12 months a number of vials are stored at $-80{ }^{\circ} \mathrm{C}$ where they are assumed to be stable. After 12 months, all standards are analyzed in one series and the average analyte concentration after each storage period and temperature is calculated.

Standards and especially working solutions are often stored in glass vials with Teflon-lined polymer screw-caps. Because the coefficient of thermal expansion of Teflon and polymers is generally much larger than that of glass, it is expected that losses due to evaporation or diffusion during storage at low temperature are minimal. Uncertainty of the compositional stability was therefore assumed to originate mainly from the evaporation of the solvent during use of the standard. The maximum amount of solvent that may be lost during the opening of the vial is the amount present in the headspace in the vial. This amount depends on the saturated vapor pressure of the solvent and can be calculated according to the following equation:

$c_{\text {solv.headspace }}=\frac{p_{\text {sat.vapor }}}{p_{\text {atm }}} \frac{M_{\text {mass }}}{M_{\text {volume }}}$

where $c$ solv.headspace is the mass concentration of the solvent in the headspace expressed in $\mathrm{mg} / \mathrm{mL}, p_{\text {sat.vapor }}$ is the solvent vapor pressure in $\mathrm{Pa}, p_{\text {atm }}$ is the atmospheric pressure in $\mathrm{Pa}, M_{\text {mass }}$ is the solvent molar mass in $\mathrm{g} / \mathrm{mol}$, and $M_{\text {volume }}$ the gaseous molar volume in $1 / \mathrm{mol}$. To determine actual losses, $100 \mathrm{~mL}$ vials, typically used for the storage of standard solutions, were filled for $50 \%$ with solvents often used in standard preparation. Losses were measured by simulating the use of these "standards" once a day for a period of 2 weeks. The vials were stored at $4{ }^{\circ} \mathrm{C}$ and allowed to warm to room temperature and mixed for $30 \mathrm{~s}$. Subsequently, the vials were opened for $1 \mathrm{~min}$ and the collection of an aliquot was simulated using a $100 \mu \mathrm{L}$ pipette, however, without actually removing or touching the liquid in the vial. After each simulation, the vials were weighed to measure the mass loss due to solvent evaporation.

\section{Results}

Uncertainty of the preparation of the analytical measurement standard

Since the measurement uncertainty depends on the uncertainty of the analytical measurement standard, it is not only necessary to know the uncertainty of this standard but also desirable (though not required) to minimize its uncertainty [16]. Therefore, when preparing the measurement standard solutions, adequate procedures and working techniques should be used to minimize the uncertainty in its analyte concentration. Use a positive displacement pipette instead of a piston operated pipette for the transfer of small volumes. Weighing may be more repeatable than volumetric 
operations although this is more labor intensive. When possible decrease the number of working steps. For the calculation of uncertainty, we considered the preparation of a measurement standard stock solution with an analyte concentration of $1000 \mathrm{mg} / \mathrm{L}$ and dilution to a measurement standard working solution with an analyte concentration of $10 \mathrm{mg} / \mathrm{L}$. Preparation of the stock solution involves weighing approximately $10 \mathrm{mg}$ of the pure compound into a tarred 25 or $50 \mathrm{~mL}$ flask, calculating the amount of solvent needed to reach the specified concentration, and adding that amount on a mass bases. This stock solution is then diluted to a working solution by volumetric dilution using pipettes and volumetric flasks. The easiest way to identify all potential sources of uncertainty is to write down the measurement function that describes the calculation of the output quantity, i.e., the final analyte mass concentration of the working measurement standard or calibrator, $c_{c}$. This analyte mass concentration is given as follows:

$c_{c}=\frac{m_{c} \cdot P_{c} \cdot \rho_{s} \cdot V_{p}}{m_{s} \cdot V_{v}}+B_{c}$

Here, $c_{c}, m_{c}$, and $P_{c}$ are the concentration of the analyte in the working calibrator, the original mass of the analyte weighed into the flask, and the fractional purity of the analyte. Further, $\rho_{s}$ and $m_{s}$ are the density and mass of solvent added to the analyte in the flask, while $V_{p}$ and $V_{v}$ are the volumes of the pipette and volumetric flask used in the preparation of the stock and working measurement standards. Finally, $B_{c}$ is the analyte mass concentration, if any, present in the blank solvent. The calculation of the uncertainties of all these input quantities is illustrated in the Eurachem/CITAC Guide [4]. The values of the input quantities described above, their standard uncertainties, and relative standard uncertainties are summarized in Table 1.

Substituting the values of the input quantity $(x)$ in Eq. 5 , results in an analyte concentration $c_{c}$ of $10.00 \mathrm{mg} / \mathrm{L}$. If $B_{c}$ can be disregarded, the measurement function in Eq. 5 involves only products and quotients and the standard uncertainties of each input quantity can simply be

Table 1 Standard uncertainties $u(x)$ and relative standard uncertainties $u(x) / x$ of input quantity $\mathrm{X}$ of the preparation of a calibrator

\begin{tabular}{llll}
\hline Input quantity $x$ & $x$ & $u(x)$ & $u(x) / x$ \\
\hline Mass compound $\left(m_{c}\right)$ & $10 \mathrm{mg}$ & $0.077 \mathrm{mg}$ & 0.0077 \\
Purity $\left(P_{c}\right)$ & 0.99 & 0.0058 & 0.0058 \\
Density $\left(\rho_{s}\right)$ & $0.789 \mathrm{~g} / \mathrm{mL}$ & $0.0026 \mathrm{~g} / \mathrm{mL}$ & 0.0033 \\
Mass solvent $\left(m_{s}\right)$ & $7.81 \mathrm{~g}$ & $0.020 \mathrm{~g}$ & 0.0026 \\
Volume pipette $\left(V_{p}\right)$ & $100 \mu \mathrm{L}$ & $1.44 \mu \mathrm{L}$ & 0.0144 \\
Volume volumetric flask $\left(V_{v}\right)$ & $10 \mathrm{~mL}$ & $0.06 \mathrm{~mL}$ & 0.0062 \\
Blank contribution $\left(B_{c}\right)$ & $0 \mathrm{~g} / \mathrm{mL}$ & $0 \mathrm{~g} / \mathrm{mL}$ & 0.0000 \\
\hline
\end{tabular}

combined, resulting in a relative standard uncertainty of 0.019 and thus a standard uncertainty of $0.19 \mathrm{mg} / \mathrm{L}$ for this $10 \mathrm{mg} / \mathrm{L}$ working measurement standard. The expanded uncertainty $U\left(c_{c}\right)$ can be calculated by multiplying the standard uncertainty with a coverage factor of 2 . This coverage factor is chosen to achieve a desired level of confidence and choosing two results in an expanded uncertainty with a confidence interval of approximately $95 \%$. For our working calibrator with a nominal concentration of $10 \mathrm{mg} / \mathrm{L}$ in methanol, the expanded uncertainty $U\left(c_{c}\right)=0.38 \mathrm{mg} / \mathrm{L}$. Major and minor contributors to the total uncertainty can be easily identified by performing all calculations in an Excel spreadsheet as described in QUAM [4] and producing the histogram shown in Fig. 1. From the histogram, it is clear that the uncertainty in $V_{p}$, the volume of the pipette is a major contributor to the total uncertainty, and that, if we want to improve the quality, e.g., decrease the uncertainty of the concentration of the working solution, we should focus our attention on $V_{p}$.

Uncertainty of the chemical stability of the analytical measurement standard

When considering the stability of measurement standards, we have to differentiate between chemical stability and compositional stability. With the former is meant changes in concentration due to chemical degradation or alteration (for example, isomerization) of the analyte. With the latter is meant changes in the concentration of the analyte due to irreversible adsorption of the analyte to the container wall, precipitation of the analyte or evaporation of the solvent. While there are a few publications dealing with the estimation of the shelf-life of reference materials [13, 17], the literature contains only little information about how to estimate the stability of home-made analytical

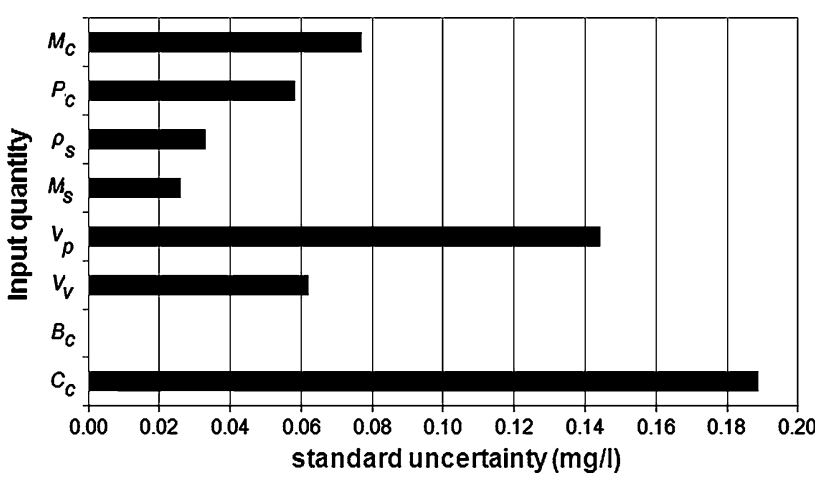

Fig. 1 Histogram showing the absolute contributions of the individual input quantities to the total uncertainty of the analytical measurement standard $\left(M_{c}\right.$ weighed mass of analyte; $P_{c}$ purity of analyte; $\rho_{s}$ density of solvent; $M_{s}$ mass of solvent; $V_{p}$ volume of the pipette; $V_{v}$ volume of the volumetric flask; $B_{c}$ concentration of the analyte in the blank; $C_{c}$ concentration of the analyte in the working calibrator) 
measurement standards, and what was found, mostly dealt with chemical stability.

Short-term stability can be measured following the Commission Decision 2002/657/EC [14]. It describes that the chemical stability of working calibrators should be tested for several weeks or longer by comparison of a stored working solution with a freshly prepared working solution. However, as a consequence of comparing working solution prepared at different dates and difference in detector response in the subsequent series relatively large measurement uncertainties will be found. This is illustrated by a study of Croubles et al. who tested the short-term stability of working calibrators for veterinary drugs [18]. Because Croubles analyzed the working calibrators in different time series, differences in detector response in each series resulted in a large measurement uncertainty. As an alternative, the organization of stability tests should be such that all samples are analyzed in one series thereby reducing potential differences in detector response and thus minimizing the resultant uncertainty $u$ (shelf).

As an example, we tested the stability of the paralytic shellfish poisons (PSP's) neosaxitoxin (NEO) and gonyautoxin (GTX-2/3), and the equivalent decarbamoyls, dc-NEO and dc-GTX-2/3. Following the preparation of the working calibrator, this was divided over a number of vials that were stored at $-20^{\circ} \mathrm{C}$. After periods of $0,1,3,6,9$, and 12 months, a number of vials were stored at $-80^{\circ} \mathrm{C}$. After 12 months, all vials were analyzed in one series, and the results are given in Table 2 and Fig. 2. The statistics in Table 2 indicate that the decrease of the dc-NEO concentration is significant at the $95 \%$ confidence level while that of the others is not significant. In relation to the organization of the stability test, it is interesting to note that the response differences in Fig. 2 appear to be small, a consequence of measuring all samples in one series. In this case, the stability uncertainty over a 1-year period ranges from $1.5 \%$ for dc-GTX-2/3 to $2.9 \%$ for dc-NEO. As can be seen in Fig. 2, the results of the measurements are in the range of $95-105 \%$ with no clear trend for NEO, GTX-2/3 and dc-GTX-2/3. While the slope of the regression line of each of these PSP's deviates from zero, $b / u(b)$ is smaller than the Student's $t$ test value indicating that this deviation is not significant since the uncertainty in the slope is much larger than the slope itself. Therefore, the working calibrators are stable for a period of at least 12 months and with $u(b)$ being the standard uncertainty of the slope calculated form the standard deviation of all measurements, the uncertainty becomes $t u_{b}$. With no change in the concentration observed over time, this expression is used to estimate $u$ (shelf) which is a linear function of $t$. This is logical because the longer one looks into the future, the less certain one is about the analyte concentration. For dc-NEO, the situation is different. The decrease in the slope is
Table 2 Chemical stability data of a calibrator for the paralytic shellfish poisons (PSP), neosaxitoxin (NEO) and gonyautoxin (GTX-2/3), and their decarbamoyl analogs dc-NEO and dc-GTX-2/3

\begin{tabular}{lllll}
\hline Storage time (month) & \multicolumn{4}{l}{ Concentration $(\mu \mathrm{g} / \mathrm{l})$} \\
\cline { 2 - 5 } & EON & dc-NEO & GTX-2/3 & dc-GTX-2/3 \\
\hline 0 & 100 & 100 & 100 & 100 \\
1 & 103 & 104 & 98 & 103 \\
3 & 100 & 97 & 99 & 101 \\
6 & 104 & 97 & 101 & 103 \\
9 & 102 & 94 & 97 & 101 \\
12 & 105 & 94 & 104 & 101 \\
\hline Slope, $b \mu \mathrm{g}(1 \mathrm{month})$ & & & & \\
Uncertainty, $u(b) \mu \mathrm{g}(1 \mathrm{month})$ & 0.153 & 0.238 & 0.215 & 0.127 \\
$|b / u(b)|$ & 2.238 & 3.049 & 1.082 & 0.317 \\
$t$ test & neg. & pos. & neg. & neg. \\
\hline
\end{tabular}

neg. = negative, pos. $=$ positive

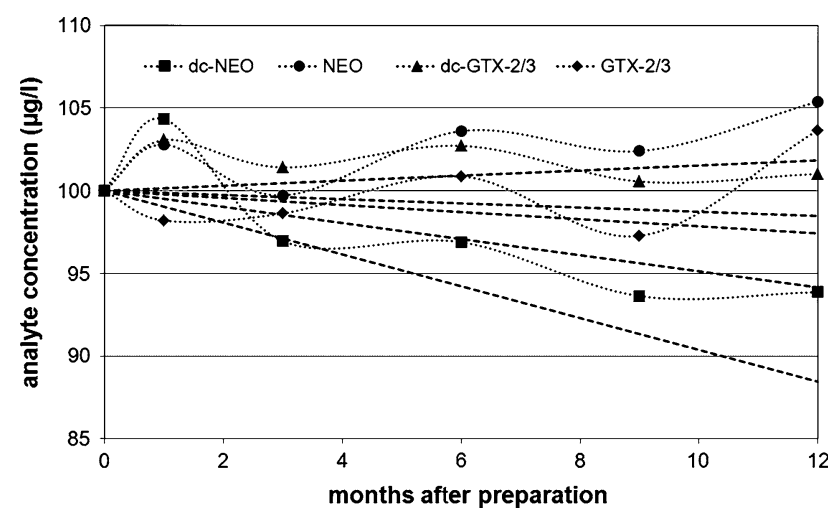

Fig. 2 Chemical stability measurement and stability uncertainty for a calibrator for the paralytic shellfish poisons (PSP), neosaxitoxin (NEO) and gonyautoxin (GTX-2/3), and their decarbamoyl analogs dc-NEO and dc-GTX-2/3 stored at $-20{ }^{\circ} \mathrm{C}$ for 12 months. Note that the curved lines only emphasize the changes in the measured concentrations as represented by the markers and do not represent real concentrations

significant, i.e., dc-NEO is degrading and the shelf-life of the standard is limited. It can be decided that this standard is simply not suitable; however, using the slope, the actual concentration over time and its uncertainty can be estimated and the standard can still be used. Additionally, if a maximum deviation for the concentration of standards is set, the slope and uncertainty data can be used to calculate the maximum shelf-life for this standard.

Uncertainty of the compositional stability

of the analytical measurement standard

For assessing the compositional stability, only the evaporation of solvents during storage or actual use of the 
Table 3 Mass values (in $\mathrm{g}$ ) for vials with different solvents stored at $-20{ }^{\circ} \mathrm{C}$ for a period up to 18 months (mean mass of empty vial was $4.66 \mathrm{~g}$ ) (neg. = negative, pos. $=$ positive $)$

\begin{tabular}{|c|c|c|c|c|}
\hline \multirow[t]{2}{*}{ Mass (g) } & \multicolumn{4}{|l|}{ Solvent } \\
\hline & Hexane & Dichloromethane & Acetonitrile & Methanol \\
\hline Maximum & 6.012 & 7.348 & 6.230 & 6.269 \\
\hline Minimum & 5.955 & 7.292 & 6.200 & 6.241 \\
\hline Mean & 5.988 & 7.324 & 6.213 & 6.257 \\
\hline Standard deviation & 0.0184 & 0.0220 & 0.0123 & 0.0117 \\
\hline Slope, $b$ g month $^{-1}$ & -0.0019 & -0.0026 & 0.0003 & 0.0009 \\
\hline Uncertainty, $u(b) \mathrm{g} \mathrm{month}^{-1}$ & 0.0009 & 0.0009 & 0.0008 & 0.0007 \\
\hline$|b / u(b)|$ & 2.23 & 2.95 & 0.37 & 1.33 \\
\hline$t$ test & neg. & pos. & neg. & neg. \\
\hline
\end{tabular}

calibrator is being considered. Long-time storage, e.g., longer than 6 months, has been tested for the solvents hexane, dichloromethane, acetonitrile, and methanol. Working calibrators with a typical volume of $2 \mathrm{~mL}$ were stored in 4-mL glass vials closed with Teflon-lined polymer screw-caps and stored in a refrigerator at $-20{ }^{\circ} \mathrm{C}$. The vials were weighed after periods of $1,2,3,612$, and 18 months. The statistics of the recorded mass values are shown in Table 3. Figure 3 shows the mass changes of all four solvents relative to $t=0$.

With the exception of dichloromethane, the results appear to be relatively randomly distributed. Since the total mass of the vials with solvent was 5-10 g and the standard uncertainty of the balance was $3 \mathrm{mg}$, a relative standard deviation of $0.3 \%$ in the seven measurements is not unrealistic. Further statistical analysis of the slope $b$ and its standard uncertainty, $u(b)$, using the $t$ test, shows that only the slope for dichloromethane is significant at a confidence level of $p=0.05$. For an 18 months period, the expected loss of dichloromethane calculated from the slope is $0.047 \mathrm{~g}$ which, based on the $2 \mathrm{~mL}$ dichloromethane in the vial, would result in an increase of the analyte concentration with $1.8 \%$. The conclusion from this observation is that dichloromethane is not a suitable solvent for calibrators that will be stored for periods of 18 months at $-20{ }^{\circ} \mathrm{C}$. The uncertainty in the composition of the working calibrator in each solvent over any period of storage can now be calculated by multiplying the standard uncertainty in the slope with the storage time. Storage of a working calibrator in hexane for a period of 12 months will lead to a compositional uncertainty of $0.18 \%$. Please note that this uncertainty is not a consequence of the evaporation of solvent, but a consequence of the uncertainty of the mass control measurements.

Since evaporation is an important contributor to the uncertainty of the compositional stability, potential solvent evaporation during actual use of the calibrator is also of importance. The maximum amount of solvent that may be lost upon opening of the vial is the amount present in the

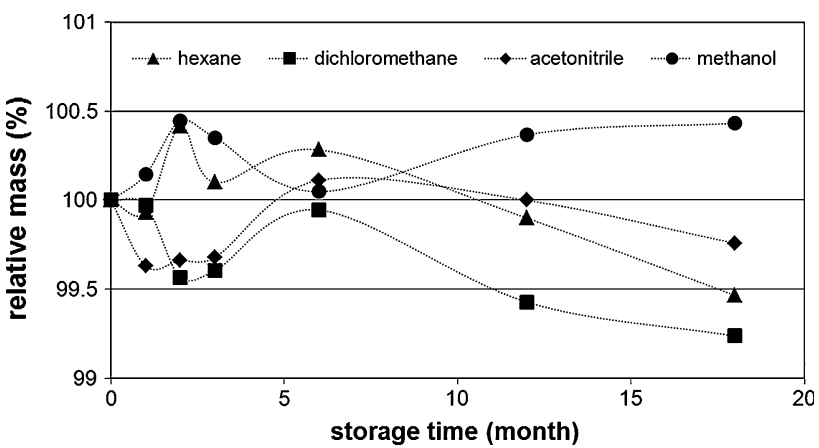

Fig. 3 Relative mass (month 0 is $100 \%$ ) of calibrators of different solvents and stored in glass vials with Teflon-lined screw-caps at $-20{ }^{\circ} \mathrm{C}$ for a period up to 18 months. Note that the curved lines only emphasize the changes in the relative mass as represented by the markers and do not represent real values

Table 4 Saturated vapor pressures (in $\mathrm{Pa}$ ) at different temperatures of a number of solvents frequently used in the preparation of calibrators for trace contaminants

\begin{tabular}{lrrrrrrr}
\hline Temperature $\left({ }^{\circ} \mathrm{C}\right)$ & -25 & -18 & -10 & -5 & 5 & 10 & 25 \\
\hline Water & 106 & 170 & 293 & 412 & 812 & 1141 & 3160 \\
Ethanol & 475 & 706 & 1108 & 1469 & 2582 & 3424 & 7980 \\
Methanol & 903 & 1340 & 2105 & 2791 & 4906 & 6505 & 15162 \\
Acetonitrile & 721 & 1070 & 1680 & 2228 & 3916 & 5193 & 12103 \\
Acetone & 2058 & 3217 & 5186 & 6880 & 11713 & 15049 & 30210 \\
\hline
\end{tabular}

headspace of the vial. This amount depends on the size of the headspace, the room temperature, and the saturated vapor pressure of the solvent. Saturated vapor pressures of a number of relevant solvents at different temperatures were collected from the literature or calculated by a method based on an article of Hass and Newton in CRC Handbook of Chemistry and Physics [19]. They are presented in Table 4.

The amount of solvent in the headspace (expressed in $\mu \mathrm{g} / \mathrm{mL}$ ) was calculated for $100 \mathrm{~mL}$ glass vials filled for $50 \%$ with solvent and using the saturated vapor pressures in Table 4. These $100 \mathrm{~mL}$ glass vials were considered 
Table 5 Mass of vials (in $\mathrm{g}$ ) with calibrators prepared in different solvents and stored at $4{ }^{\circ} \mathrm{C}$ for a period up to 14 days (average mass of empty vials was $22.51 \mathrm{~g}$ )

\begin{tabular}{llllll}
\hline Mass $(\mathrm{g})$ & \multicolumn{3}{l}{ Solvent } & & \\
\cline { 2 - 6 } & Acetonitrile & Ethanol & Acetone & Methanol & Water \\
\hline Maximum & 61.958 & 62.139 & 62.428 & 62.011 & 68.327 \\
Minimum & 61.900 & 62.099 & 62.245 & 61.952 & 68.310 \\
Mean & 61.930 & 62.118 & 62.337 & 61.981 & 68.317 \\
Standard deviation & 0.0189 & 0.0130 & 0.0594 & 0.0192 & 0.0050 \\
\hline Slope, $b$ g month $^{-1}$ & -0.0045 & -0.0031 & -0.0142 & -0.0046 & -0.0012 \\
Uncertainty, $u(b) \mathrm{g} \mathrm{month}^{-1}$ & 0.00010 & 0.00007 & 0.00010 & 0.00007 & 0.00007 \\
$|b / u(b)|$ & 44 & 43 & 143 & 70 & 16 \\
$t$ test & pos. & pos. & pos. & pos. & pos. \\
\hline
\end{tabular}

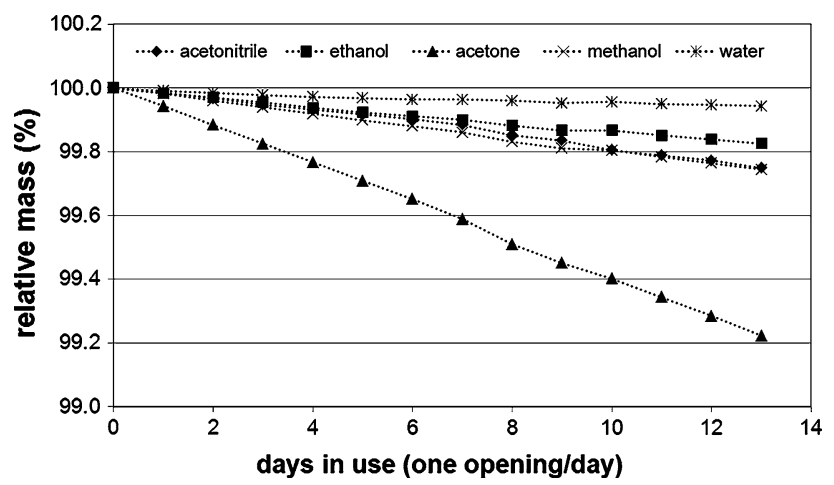

Fig. 4 Relative mass (day 0 is 100\%) of calibrator of different solvents and stored in glass vials with Teflon-lined screw-caps at $4{ }^{\circ} \mathrm{C}$ for a period of 14 days

typical for the storage of working calibrators while 50\% was taken as an average. The loss in mass due to evaporation at $25{ }^{\circ} \mathrm{C}$ are $0.6 \mathrm{mg}$ for water, $4.4 \mathrm{mg}$ for ethanol, $5.8 \mathrm{mg}$ for methanol, $5.9 \mathrm{mg}$ for acetonitrile, and $22 \mathrm{mg}$ for acetone. In addition to these calculations, experiments were carried out to measure the mass losses every day during a 14 day period in the same type of vials stored at $4{ }^{\circ} \mathrm{C}$. Before use they were allowed to warm to room temperature and the content mixed for $30 \mathrm{~s}$. Subsequently, the vials were opened for $1 \mathrm{~min}$ and the collection of a number of aliquots was simulated using a $100 \mu \mathrm{L}$ pipette, however, without actually removing or even touching the liquid in the vial. After each simulation, the vials were closed and weighed to determine the loss in mass due to solvent evaporation. The statistics of the recorded mass values are presented in Table 5. Figure 4 shows the change in mass of the vials relative to the mass at $t=0$.

The measurement results in Fig. 4 show a very regular decrease in mass for all solvents as indicated by the high significance of the slope in Table 5. Comparison of the experimental mass values to the theoretical maximum loss of solvent in the headspace of the vials shows that the actual loss is about $70 \%$ of the maximum possible.
Table 6 Contributions to the total measurement uncertainty of the calculated value in the calibrator

\begin{tabular}{ll}
\hline Description & $u(x) / x$ \\
\hline Uncertainty due to preparation & $1.9 \%$ \\
Uncertainty in chemical stability (average of three & $2.0 \%$ year $^{-1}$ \\
PSP compounds, excluding the non-stable & \\
dc-NEO) & \\
Uncertainty in long-term compositional stability & $0.53 \%$ year $^{-1}$ \\
$\quad($ methanol, long-term storage calibrator stock at & \\
$\left.\quad-20^{\circ} \mathrm{C}\right)$ & \\
Uncertainty in short-term compositional stability & $0.35 \%$ month $^{-1}$ \\
$\quad$ (methanol, use and short-term storage working & \\
calibrator at $4{ }^{\circ} \mathrm{C}$ ) & \\
\hline
\end{tabular}

\section{Discussion}

The total uncertainty of the concentration in a chemical calibrator depends on the uncertainty of the preparation and the uncertainties of the chemical and compositional stability. As an illustration, the average value of the relative standard uncertainties of the concentrations of the three stable PSP analytes in the PSP calibrator in this study is given in Table 6. Note that the uncertainty in the chemical and compositional stability is expressed per unit of time. The values for the chemical and compositional stability are calculated by multiplying the uncertainty in the slope $u(b)$ with time $t$ when the slope is not significant (chemical stability of the three stable PSP analytes and long-term stability of methanol). When the slope is significant (degradation of dc-NEO and short-term stability of all solvents), the uncertainty should be calculated by multiplying the uncertainty in the slope $u(b)$ with time $t$, and by multiplying the slope $b$ itself with time $t$ to correct for the real degradation. The two contributions should be added quadratically. For the short time compositional stability, it is assumed that the standard is used each day which is, of course, a worst case scenario. 


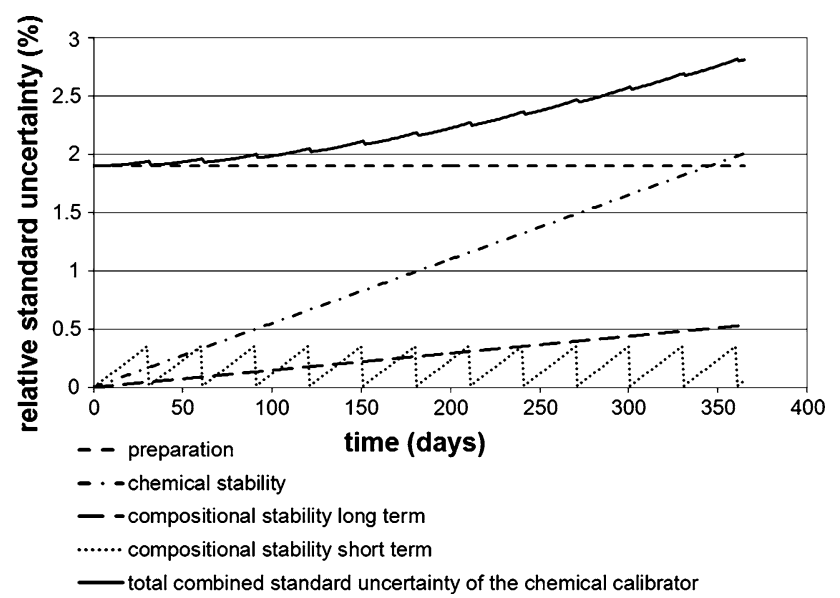

Fig. 5 Visual presentation of the contributions of preparation and stability uncertainties to the total combined standard uncertainty of a chemical calibrator in time

Considering a storage time of 1 year for the calibrator stock and 1 month for the working calibrator, it follows that the maximum total combined standard uncertainty is $2.8 \%$. For a working calibrator of $10 \mathrm{mg} / \mathrm{L}$, the standard uncertainty becomes $0.28 \mathrm{mg} / \mathrm{L}$ and the expanded uncertainty with a coverage factor of 2 (95\% confidence interval) will be $0.57 \mathrm{mg} / \mathrm{L}$ for the storage periods mentioned above. The increase in the uncertainties is graphically expressed in Fig. 5 where it is considered that working calibrators stored at $4{ }^{\circ} \mathrm{C}$ are prepared fresh from the calibrator stock every month. From this it is clear that the preparation of the chemical calibrator and (in time) the chemical stability contribute most to the total uncertainty of the value carried by the calibrator. However, also notice that the compositional stability of the working calibrators stored at $4{ }^{\circ} \mathrm{C}$ may become a major source if these are not renewed frequently and are used for periods exceeding 3 months.

\section{Conclusions}

This study describes a general procedure to evaluate and calculate the uncertainty of the values embodied in the chemical calibrators and the changes in the uncertainty of their actual analyte concentration over time. In general, the preparation procedure and the chemical stability will be the major sources of their total uncertainty. Note that the uncertainty in the stability mostly results from the uncertainties in the stability measurements themselves and not from actual instability of the analytes in the analytical standard. With respect to the latter conclusion, it was shown that the procedure of the stability measurements is of importance to limit the stability uncertainty. Of course it should be noted that the results in this study only apply to the preparation procedure and materials used in this study. For other procedures or materials, the uncertainties will have to be re-evaluated and recalculated. However, the calculation methods shown will be useful for the evaluation of total uncertainties and the identification of the major individual sources contributing to these uncertainties.

Open Access This article is distributed under the terms of the Creative Commons Attribution Noncommercial License which permits any noncommercial use, distribution, and reproduction in any medium, provided the original author(s) and source are credited.

\section{References}

1. ISO/IEC 17025 (2005) General requirements for the competence of testing and calibration. ISO, Geneva, Switzerland

2. BIPM, IEC, IFCC, ISO, IUPAC, IUPAP, OIML (2008) Guide to the expression of uncertainty in measurement. JCGM 100. http://www.bipm.org/en/publications/guides/gum.html

3. ISO 21748 (2010) Guidance for the use of repeatability, reproducibility and trueness estimates in measurement uncertainty estimation. ISO, Geneva, Switzerland

4. Ellison SLR, Rösslein M, Williams A (eds) (2000) Eurachem/ CITAC Guide; Quantifying uncertainty in analytical measurement. Eurachem, 2nd edn

5. Ellison SLR, Williams A (eds) (2007) Eurachem/CITAC Guide, use of uncertainty information in compliance assessment. Eurachem, 1st edn

6. Populaire S, Campos Gimenez E (2006) Accred Qual Assur 10:485-493

7. Maroto A, Boque R, Vander Heyden Y (2008) LC/GC Europe 21:628-631

8. European Federation of National Associations of Measurement, Testing and Analytical Laboratories (EuroLab) (2007) Measurement uncertainty revisited: alternative approaches to uncertainty evaluation. Technical Report No. 1/2007, March 2007

9. Armishaw P (2003) Accred Qual Assur 8:218-224

10. Jurado JM, Alcázar A (2005) Accred Qual Assur 10:373-381

11. BIPM, IEC, IFCC, ISO, IUPAC, IUPAP, OIML (2008) International vocabulary of metrology - basic and general concepts and associated terms (VIM), 3rd edn. JCGM 200

12. De Bièvre P (2008) Metrologia 45:335-341

13. Linsinger TPJ, Pauwels J, Lamberty A, Schimmel HG, van der Veen AMH, Siekmann L (2001) Fresenius J Anal Chem 370: $183-188$

14. Commission Decision 2002/657/EC (2002) Implementation of Council Directive 96/23/EC concerning the performance of analytical methods and the interpretation of results. Official Journal of the European Communities, L 221

15. Lamberty A, Schimmel H, Pauwels J (1998) Fresenius J Anal Chem 360:359-361

16. Meyer VR, Majors RE (2002) LC/GC Europe 15:2-5

17. Sarembaud JM, Feinberg M (2007) Accred Qual Assur 12:75-83

18. Croubels S, De Baere S, De Backer P (2003) Anal Chim Acta 483:419-427

19. Hass HB, Newton RF, Weast RC (eds) (1971) Handbook of chemistry and physics. D. The Chemical Rubber Co., Cleveland, pp 144-145 